\title{
Introduction à la thématique spéciale portant sur le combat contre le racisme anti-noir dans la formation donnée en sciences, en mathématiques, en technologie et dans les STIM
}

\author{
Fikile Nxumalo $^{1} \cdot$ Wanja Gitari $^{1}$
}

Accepted: 8 July 2021 / Published online: 24 July 2021

(C) Ontario Institute for Studies in Education (OISE) 2021

La pandémie de la COVID-19 a fait ressortir une nouvelle fois les impacts des injustices qui touchent l'ensemble des aspects liés à la race, aux genres et aux classes sociales. La pandémie fait ressortir encore plus les inégalités systémiques actuelles dans le domaine de la santé et leur effet brutal sur les personnes noires (Etowa et Hyman, 2021). Parallèlement, une montée de l'opposition mondiale face à la violence faite aux Noirs, après les meurtres de Breonna Taylor, d'Ahmaud Arbery et de George Floyd a amplifié une prise en compte de la réalité quant aux effets actuels liés à «l'état d'urgence mondiale face au racisme anti-noir» (Brand, 2020, paragr. 1) manifesté sous toutes ses formes les plus tenaces et toutes ses violences. Ce moment de vérité a mené à un questionnement face au racisme anti-noir dans le domaine des sciences, comme en font foi les mouvements et groupes qui sont apparus sur les réseaux sociaux comme \#BlackinNeuro et \#ShutDownSTEM (Chen, 2020; Ortolano, 2020). Motivés par le potentiel déstabilisant de la période que l'on vit, les articles publiés sous ce thème spécial proposent de considérer certaines pistes de solutions en ce qui a trait à la formation donnée en sciences, en mathématiques, en technologie et dans les STIM afin de favoriser des pratiques libératrices et des orientations conceptuelles qui tendent à briser le cycle de la logique anti-noire.

Le racisme anti-noir, «indissociable de l'esclavage racial et de ce qui en résulte, demeure la condition antisociale extrême en ce qui concerne la possibilité du monde social moderne» (Costa Vargas et Jung, 2021, p. 4). Nous commençons cette thématique spéciale en pointant le racisme anti-noir pour souligner que l'élément central du combat soulevé par le moment que nous vivons consiste à contrer la logique anti-noire - ce qui constitue une réponse distincte de celle globalement donnée au racisme en général. Costa Vargas et Jung (2021) fournissent une conceptualisation profitable du racisme anti-noir:

Un cadre de racisme anti-noir met en valeur l'unicité du positionnement et des expériences des Noirs par rapport aux groupes sociaux qui ne sont pas noirs. Ce cadre propose une définition de l'antagonisme de la modernité qui met en opposition les Noirs et les non-Noirs (Wilderson, 2010).

Fikile Nxumalo

f.nxumalo@utoronto.ca

Wanja Gitari

wanja.gitari@utoronto.ca

1 Ontario Institute for Studies in Education, University of Toronto, Toronto, ON M5S 1V6, Canada 
Le résultat d'efforts théoriques et d'analyses historiques et sociologiques, une telle perspective laisse entendre que les personnes noires (a) sont non seulement exceptionnellement et systématiquement exclues socialement - des marchés résidentiels, d'une éducation de qualité, des soins de santé efficaces, d'une garantie de sécurité et de vie - mais aussi (b) qu'elles représentent un «non-être » qui sous-tend et engendre les subjectivités modernes des non-Noirs. Ces propositions adoptent une logique d'abjection sociale et ontologique, plutôt que de domination ou de soumission des personnes noires. Une telle logique constitue le racisme anti-noir. (p. 7-8)

Il existe un corpus de plus en plus nombreux d'études savantes en éducation qui porte sur le racisme anti-noir en tant que cadre dans le but de déterminer comment l'enseignement scolaire constitue un lieu de souffrance et de marginalisation pour les jeunes et les enfants noirs (Dumas et Ross, 2016; Nxumalo et Ross, 2019; Warren, 2021). Cela comprend des études qui ont démontré que la formation dans les sciences, les mathématiques, la technologie et les STIM continue d'être un milieu où la logique du racisme anti-noir persiste (Bullock, 2017; Cedillo, 2018; Martin, Price et Moore, 2019; Vakil et Ayers, 2019).

Les effets cumulatifs du racisme anti-noir dans l'espace réservé aux sciences, aux mathématiques, à la technologie et aux STIM sont ressentis de multiples façons. Cela se traduit par la sous-représentation d'étudiants noirs dans les domaines des STIM (Morton et al., 2019; Riegle-Crumb et al., 2019). L'on constate aussi des effets dans les domaines STIM relatifs à la technologie tels que la formation donnée en informatique où le racisme anti-noir se manifeste par des lacunes persistantes en ce qui a trait aux chances des étudiants noirs d'avoir accès à la technologie et aux cours avancés (Madkins et al., 2020).

Dans les salles de cours de mathématiques, Martin et ses collaborateurs (2019) ont signalé les raisons pour lesquelles la violence symbolique et épistémique contre les Noirs persiste à travers la normalisation des discours racisés sur l'absence de réussite; par le truchement d'expressions pédagogiques qui positionnent les mathématiques comme étant le domaine des hommes de race blanche et d'origine asiatique; par le biais de pratiques de suivi anti-noires; et, à travers des exemples de programmes d'études anti-noirs. La violence épistémique s'étend au-delà des milieux des mathématiques et comprend les façons dont les épistémologies scientifiques qui ne sont pas euro-occidentales sont continuellement marginalisées ou entièrement supprimées (Bang et Medin, 2010). À cet égard, la formation en sciences, en mathématiques, en technologie et dans les STIM pérennise à la fois le racisme anti-noir et la «colonialité» (Vakil et Ayers, 2019; Warren et collab., 2020). Le racisme anti-noir peut aussi prendre plusieurs formes subtiles - y compris celles dites progressistes. Par exemple, le fait de porter une attention particulière aux personnalités noires qui ont marqué l'histoire de l'enseignement du génie efface non seulement la participation et les succès actuels des personnes noires en ingénierie, mais peut aussi teinter le discours portant sur les succès des Noirs dans le domaine en dépeignant ces réussites comme une exception plutôt que la norme (Holly, 2020).

La logique anti-noire demeure présente en dépit des initiatives visant à renforcer l'équité, la diversité et l'inclusion dans les salles de cours en sciences, en mathématiques, en technologie et en STIM (McGee et collab., 2021). Par exemple, malgré les réformes orientées vers l'inclusion, les salles de classe en mathématiques continuent d'être des lieux de non-appartenance pour les étudiants noirs, comme on peut le voir quand l'inclusion s'avère assimilatrice et qu'elle a peu d'impact sur les cultures dominantes néfastes dans l'enseignement des mathématiques (Martin, 2019). Également, il a été démontré que des initiatives comme celles d'implanter des académies de STIM dans les écoles urbaines défaillantes exacerbent l'iniquité éducationnelle racisée et contribuent au déplacement des étudiants noirs; le maintien des processus fondés sur le mérite, exclusifs; et l'érection et la préservation d'une formation en STIM qui est le propre des Blancs (Bullock, 2017). Cela ne veut pas dire que les efforts d'inclusion sont nécessairement mauvais. À l'opposé, comme démontré dans les articles publiés dans ce numéro, l'inclusion comporte un potentiel transformateur. Néanmoins, il importe de complexifier les discours d'inclusion qui sont exempts de critiques dans les domaines des STIM. Ceci comprend la nécessité de signaler les tensions inhérentes au fait d'intégrer les étudiants noirs aux 
domaines des STIM et qui entretiennent l' «extrativisme» planétaire, le militarisme et le capitalisme racial (Nxumalo et Ross, 2019; Vakil et Ayers, 2019; Vossoughi et Vakil, 2018).

Il n'y a que trop d'exemples que nous pouvons soulever pour parler des façons dont le racisme antinoir perdure et se renouvelle dans les contextes d'enseignement des sciences, des mathématiques, de la technologie et des STIM. Dans ce numéro, en adoptant une orientation qui prend au sérieux les limites de la promotion du changement, et en mettant l'accent sur les récits de préjudice et leur diffusion (Tuck, 2009), nous nous intéressons aux possibilités de transformation des relations de pouvoir inéquitables. Dans la mesure où l'on réaliserait et concevrait autrement le domaine des sciences, des mathématiques, de la technologie et des STIM, les articles qui se retrouvent dans cette thématique spéciale tentent collectivement de déterminer si le refus du racisme anti-noir permettrait des conceptualisations plus positives de l'avenir des Noirs. Dans l'appel fait en appui à cette thématique, nous étions particulièrement intéressés aux possibilités de transformer l'éducation afin qu'elle devienne un milieu favorable à l'élaboration et à la conception d'un futur pour les Noirs (Nxumalo, 2021; Warren, 2021). Ceci s'inscrit dans la foulée de nos remarques préliminaires sur les impacts disproportionnés de la pandémie et sur la prise de conscience mondiale face à la violence faite aux Noirs qui amplifient l'importance du moment de vérité que nous vivons ainsi que son potentiel pour transformer alors que nous nous éloignons des systèmes en place et des pratiques qui, entre autres injustices ont normalisé le racisme anti-noir, l'individualisme, et notre façon de vivre avec le monde naturel fondé sur l'extraction de ses ressources (Bang, 2020; Benjamin, 2020). Comme la poétesse canadienne Dionne Brand (2020) l'a si bien dit, il est maintenant temps de refuser un retour à « une normale affreuse qui est narrativisée en tant qu'une série de petites injustices» (paragr. 2).

Les approches orientées vers la justice qui sont adoptées dans les articles publiés sous cette thématique spéciale en réponse à la dynamique actuelle sont variées et comprennent une focalisation sur les programmes d'études et la pédagogie, des initiatives qui mettent en valeur l'équité et l'inclusion, et des mesures conceptuelles pour briser le cycle du racisme anti-noir. De plus, s'inscrivant dans le cadre général mettant l'accent sur le combat contre le racisme anti-noir, les articles avancent plusieurs orientations théoriques, dont la «Théorie critique de la race» (Burke et al., 2021; la clarté politique (Madkins et Morton, 2021); la «hantologie» (Motala et Stewart, 2021); et la «théorie critique concernant les Noirs» (Jones et Melo, 2021). Cette multitude de perspectives et d'approches est importante — le racisme anti-noir apparait de manière complexe et à des niveaux multiples dans des contextes éducatifs spécifiques des domaines des sciences, des mathématiques, de la technologie et des STIM. Le racisme anti-noir est «fulgurant innovateur, même viral... [il] déforme la réalité, sur les plans interpersonnel et institutionnel» (Benjamin, 2020, paragr. 12-13). Le racisme anti-noir se renouvelle constamment, même sous le couvert du «progrès» et de l'inclusion et conséquemment, il faut mettre de l'avant des mesures qui sont nombreuses et contextualisées, créatives et imaginatives et qui contribuent à l'avenir des Noirs.

Nous tenons aussi à souligner que les STIM sont abordées de différentes façons et dans un sens élargi dans cette thématique spéciale. Ceci implique d'interagir avec le domaine des STIM de manière à interpeller une à une ses disciplines, telles que les sciences, l'informatique, le génie, les mathématiques et la médecine. Sur le plan interdisciplinaire, nous faisons aussi un usage plus large de l'expression «STIM» en ce qui concerne le champ des disciplines associées aux sciences. Les auteurs ciblent aussi les STIM pour discuter de l'intégration de différentes disciplines des STIM au sein des programmes de la maternelle à la $12^{\mathrm{e}}$ année. Ces divers engagements soulignent les aspects social, politique, et culturel des contextes situationnels issus du paysage complexe que constitue la formation en STIM. Les sens donnés aux STIM et à son enseignement sont abordés de manière à bouleverser les approches universalistes et décontextualisées qui distinguent l'épistémologie de l'ontologie et qui clament un point de vue «objectif» transcendant qui vient de nulle part (Haraway, 2003; Motala et Stewart, 2021; Vakil et Ayers, 2019).

Les articles publiés dans cette thématique spéciale reproduisent aussi des engagements contextualisés avec la subjectivité noire. Alors que le racisme anti-noir constitue un cadre important pour 
comprendre comment les systèmes et les pratiques qui visent à déshumaniser et exclure se manifestent dans une multitude de contextes en ce qui concerne ceux qui sont collectivement racisés en tant que Noirs, il ne faut tout de même pas concevoir de façon monolithique ce qu'il en est d'être noir. Par exemple, dans leur article, Madkins et Morton (2021) soulignent l'importance de s'attarder aux manières dont l'identité ethnique se manifeste dans l'expérience des étudiants noirs et face au racisme anti-noir auquel ils sont confrontés. Madkins et Morton indiquent que, dans le contexte de leur travail aux États-Unis, cette démarche implique de s'arrimer aux spécificités des étudiants qui peuvent se désigner eux-mêmes comme afro-américains, noirs latino-américains, indigènes noirs, antillais et immigrés en provenance de pays d'Afrique, parmi plusieurs autres marqueurs d'identité ethnique. Les articles de ce numéro s'attardent collectivement aux complexités situationnelles de la subjectivité noire à l'intérieur et au-delà des limites des États-nations, incluant sans s'y limiter les étudiants noirs canadiens en médecine (Saddler et collab., 2021), les étudiants universitaires noirs sud-africains et canadiens en génie (Burke et al., 2021; Motala et Stewart, 2021), et aux États-Unis, en informatique (Jones et Melo, 2021). Surtout, la subjectivité noire est perçue comme étant continuellement en mouvement; en processus de négociation placée en relation avec l'histoire, les discours, les expériences intersectionnelles particulières dans les STIM et les disciplines associées, et plus encore (Wright, 2004).

Globalement, les cinq articles de cette thématique spéciale adoptent une multitude de points de vue privilégiés pouvant influencer, faire ressortir et briser le cycle du racisme anti-noir; y compris des possibilités de participation à la formation donnée dans les sciences, les mathématiques, la technologie et les STIM afin qu'elle s'aligne avec des engagements libérateurs, abolitionnistes et orientés vers l'équité et qui font échec aux structures oppressives.

Dans le contexte de l'enseignement de l'informatique aux États-Unis, l'article de Jones et Melo (2021) propose aussi des possibilités libératrices. Les auteurs ciblent de façon convaincante la fiction spéculative afin de rendre apparent le racisme anti-noir inhérent au domaine de l'informatique et de permettre d'imaginer des possibilités abolitionnistes, anticoloniales et réparatrices. S'inspirant de Ruha Benjamin (2016), leur travail permet à la fois de déterminer quels sont les rouages du racisme antinoir dans l'enseignement de l'informatique et de reproduire une narration spéculative qui «réinvente et reconçoit tout ce qui est acquis sur la structure actuelle du monde social» (p. 2). Puisant dans leurs expériences personnelles de racisme anti-noir dans l'enseignement de l'informatique, Jones et Melo proposent des témoignages affectifs, incarnés et contextualisés de ce que cela pourrait signifier pour les étudiants noirs, non seulement de survivre aux conditions de racisme anti-noir en informatique, mais aussi de s'épanouir dans une ère de «joie noire». Surtout, Jones et Melo présentent une vision de l'enseignement de l'informatique et d'autres «créatures» technologiques qui soutiennent des relations durables avec la terre plutôt que celles qui sont fondées sur l'extraction de ses ressources; une orientation cruciale à l'ère des dommages anthropiques à l'échelle planétaire.

Madkins et Morton (2021) s'intéressent aux possibilités de faire échec au racisme anti-noir et à la «colonialité» en s'engageant dans des possibilités de faire autrement dans les pédagogies utilisées pour la formation de base des enseignants en STIM. Cet article, qui s'inscrit dans la thématique spéciale s'appuie sur un engagement pédagogique de clarté politique qui exige des candidats à l'enseignement qu'ils s'engagent à bien comprendre les rouages et les impacts des iniquités structurelles et scolaires. Madkins et Morton proposent des stratégies efficaces et concrètes que les candidats à l'enseignement peuvent utiliser pour bien saisir les réalités vécues par les Noirs et les indigènes et pour implanter des approches de justice sociale. Ces stratégies, élaborées pour briser le cycle du racisme anti-noir, comprennent des éléments de conception spécifiques aux salles de classe; des structures participatives comme des méthodes servant à cerner les pratiques culturelles changeantes des étudiants et leurs expériences vécues; ainsi que les modes utilisés pour établir des liens explicites entre le contenu véhiculé dans les STIM et 
l'injustice sociale. Surtout, cet article décrit des manières pour les enseignants de contrer les discours dominants de non-appartenance qui nuisent aux jeunes apprenants noir et indigènes dans les STIM.

Nous tournant vers des contextes postsecondaires, dans «Des apparitions qui transcendent le clivage: l'activisme transdisciplinaire, les dualismes et les spectres du racisme dans la formation en ingénierie ainsi qu'en lettres et sciences humaines», Motala et Stewart (2021) se penchent aussi sur les possibilités de faire autrement dans les engagements pédagogiques qui brisent le cycle du racisme anti-noir et de la «colonialité». À partir des contextes sud-africain et états-unien, les auteurs racontent les possibilités d'interruption qui ont fait surface lorsque des étudiants universitaires en ingénierie dans un cours de systèmes d'information géographique en Afrique du Sud se sont investis dans l'apprentissage avec des étudiants d'un cours de rédaction aux États-Unis en collaborant par l'entremise de groupes de clavardage sur «WhatsApp». Se penchant sur des analyses post-humanistes effectuées de façon «diffractive» sur des dialogues et artéfacts tirés de cours, les auteurs font ressortir à la fois les potentialités disciplinaires et les ingénieries pédagogiques transnationales. Avec le concept de «hantologie» à l'esprit, les auteurs rendent aussi visibles les défis associés à l'adoption de pédagogies transformatrices dans un contexte mondial permanent de racisme anti-noir et antimusulman ainsi que de dualismes coloniaux.

Burke et al. (2021) se penchent aussi sur la discipline du génie. Leur article traite des efforts faits par la Faculté des sciences appliquées et de génie (FSAG) de l'Université de Toronto (U de T) pour freiner le racisme anti-noir. Parmi les initiatives multidimensionnelles mises en place se trouvent des activités de rayonnement ciblant les niveaux de la maternelle à la $12^{\mathrm{e}}$ année, la collecte de données fondées sur la race et la création d'occasions favorisant un apprentissage centré sur la race. Par la «Théorie critique de la race» (TCR) et des récits personnels, l'article montre l'ampleur des défis rattachés au désir de changement quant au racisme anti-noir compte tenu des obstacles tels que l'absence d'engagements financiers institutionnels visant à soutenir des activités de sensibilisation axées sur la communauté qui sont durables, la réticence à rendre visible et confronter la «blanchitude» dans la discipline du génie à la FSAG de l'U de T, et la persistance de discours méritocratiques en ingénierie. Tout en admettant la complexité de la tâche liée à l'élimination des obstacles, les auteurs proposent des moyens importants pour y arriver. Par exemple, ils soulèvent l'importance d'obtenir du soutien institutionnel durable pour le travail fait par le bureau pour le rayonnement étudiant en ingénierie afin qu'il puisse continuer à cibler les partenaires communautaires qui peuvent en retour offrir du soutien aux étudiants noirs. Ce travail montre aussi l'urgence pour les facultés de génie au Canada d'accélérer la collecte de données fondées sur la race et de passer à l'action. Tel que mentionné dans l'article, le manque relatif de collecte de données fondées sur la race demeure un obstacle important qui s'étend au-delà de l'Université de Toronto et qui touche tous les établissements d'enseignement postsecondaires.

L'article de Saddler et ses collaborateurs s'intéresse aussi aux enjeux d'exclusion basée sur le racisme anti-noir à l'Université de Toronto; plus particulièrement à la faculté de médecine Temerty. Soulignant les effets du racisme systémique dans les établissements de soins de santé sur les piètres conditions de santé des communautés noires au Canada, les auteurs mentionnent que la concordance ethnique entre le patient et le médecin demeure un aspect prioritaire pour éliminer les iniquités en soins de santé dans les communautés noires. En abordant le sujet du besoin urgent de médecins noirs au Canada, l'article s'attarde aux récentes initiatives lancées par la faculté de médecine Temerty de l'Université de Toronto; à savoir des programmes de mentorat qui fonctionnent dans le cadre d'un programme de demandes pour les étudiants noirs. Les auteurs mentionnent l'efficacité de ces programmes, non seulement pour améliorer la représentation des étudiants noirs en médecine, mais aussi en matière d'impact sur les communautés noires environnantes. Le programme de demandes promeut la participation des communautés noires de la région métropolitaine de Toronto à l'intérieur et au-delà des domaines de la santé en soutien aux processus d'admission pour les étudiants noirs. Surtout, les auteurs indiquent que l'inclusion des candidats noirs dans les domaines médicaux n'est qu'une étape nécessaire — ils soulignent par ailleurs le travail actuel fait pour s'attaquer aux enjeux d'équité et au racisme anti-noir dans les programmes 
d'études des facultés de médecine et pour soutenir les expériences des étudiants noirs dans les facultés de médecine. Une part importante de ces efforts consiste à fournir aux étudiants noirs en médecine des occasions de rayonnement qui les relient aux communautés noires.

Un an après le soulèvement mondial contre le racisme anti-noir, nous nous trouvons toujours au tournant d'une période «de potentialités radicales de ce moment historique remarquable [...] un "phénix d'espoir" porteur de rêves revigorés de liberté et de résistance pour les Noirs» (Ross, 2021, p. 229). Chacun des articles s'attarde sur les possibilités transformatrices du moment que nous vivons dans différents contextes reliés à l'enseignement des sciences, des mathématiques, de la technologie et des STIM. Alors que les articles font ressortir les problèmes persistants associés au démantèlement de la logique anti-noire, ils offrent des possibilités inspirantes pour accéder à un avenir plus libérateur dans la formation donnée en sciences, en mathématiques, en technologie et dans les STIM.

\section{Références}

Bang, M. (2020). Learning on the move toward just, sustainable, and culturally thriving futures. Cognition and Instruction, 38(3), 434-444. https://doi.org/10.1080/07370008.2020.1777999

Bang, M., \& Medin, D. (2010). Cultural processes in science education: Supporting the navigation of multiple epistemologies. Science Education, 94(6), 1008-1026.

Benjamin, R. (2016). Racial fictions, biological facts: Expanding the sociological imagination through speculative methods. Catalyst: Feminism, Theory, Technoscience, 2(2), 1-28.

Benjamin, R. (2020). Black skin, white masks: Racism, vulnerability \& refuting Black pathology. Retrieved from: https:// aas.princeton.edu/news/black-skin-white-masks-racism-vulnerability-refuting-black-pathology

Brand, D. (2020). On narrative, reckoning and the calculus of living and dying. Toronto Star. Retrieved from https:// www.thestar.com/entertainment/books/2020/07/04/dionne-brand-on-narrative-reckoning-and-the-calculus-of-living-and-dying.html

Bullock, E. C. (2017). Only STEM can save us? Examining race, place, and STEM education as property, Educational Studies, 53(6), 628-641.

Burke, M., Hanson, C. \& Abraham, C. (2021). Addressing Black inclusivity within a Canadian post-secondary engineering faculty: A critical perspective. Canadian Journal of Science, Mathematics, and Technology Education. https:// doi.org/10.1007/s42330-021-00155-5

Cedillo, S. (2018). Beyond inquiry: Towards the specificity of anti-Blackness studies in STEM education. Canadian Journal of Science, Mathematics, and Technology Education, 18, 242-256.

Chen, S. (2020). Researchers around the world prepare to \#ShutDownSTEM and 'Strike For Black Lives'. Retrieved from: https://www.sciencemag.org/news/2020/06/researchers-around-world-prepare-shutdownstem-and-strike-black-lives

Costa Vargas, J. H. \& Jung, M-K. (2021). Introduction: Antiblackness of the social and the human. In Jung, M-K \& J.H. Costa (Eds.), Antiblackness (pp. 1-14). Duke University Press.

Dumas, M. J., \& Ross, K. M. (2016). "Be real Black for me”: Imagining BlackCrit in education. Urban Education, 51(4), $415-442$.

Etowa, J., Hyman, I. (2021) Unpacking the health and social consequences of COVID-19 through a race, migration and gender lens. Can J Public Health 112, 8-11. https://doi.org/10.17269/s41997-020-00456-6

Haraway, D. (2003). Situated knowledges: The science question in feminism and the privilege of partial perspective. In Y. S. Lincoln \& N. K. Denzin (Eds.) Turning points in qualitative research: Tying knots in a handkerchief (pp.21-46). Lanham: Altamira Press,

Holly, J. Jr. (2020). Disentangling engineering education research's anti-Blackness. Journal of Engineering Education, 109(4), 629-635. https://doi.org/10.1002/jee.20364

Jones, S.T. \& Melo, N.A. (2021). We tell these stories to survive: Towards abolition in computer science education. Canadian Journal of Science, Mathematics, and Technology Education. https://doi.org/10.1007/s42330-021-00158-2

Madkins, T. C. \& Morton, K. (2021). Disrupting anti-Blackness with young learners in STEM: strategies for elementary science and math teacher education. Canadian Journal of Science, Mathematics, and Technology Education. https:// doi.org/10.1007/s42330-021-00159-1

Madkins, T. C., Howard, N. R., \& Freed, N. (2020). Engaging Equity Pedagogies in Computer Science Learning Environments. Journal of Computer Science Integration, 3(2), 1-27.

Martin, D. B. (2019). Equity, inclusion, and antiblackness in mathematics education. Race Ethnicity and Education, 22(4), 459-478. 
Martin, D. B., Price, P. G., \& Moore, R. (2019). Refusing systemic violence against Black children: Toward a Black liberatory mathematics education. In J. Davis \& C.C. Jett (Eds.), Critical race theory in mathematics education (pp. 32-55). Routledge.

McGee, E. O. Botchway, P. K., Naphan-Kingery, D. E., Brockman, A. J., Houston II, S. \& White, D.T. (2021). Racism camouflaged as impostorism and the impact on black STEM doctoral students. Race Ethnicity and Education. https:// doi.org/10.1080/13613324.2021.1924137

Morton, T. R., Gee, D. S., \& Woodson, A. N. (2019). Being vs. becoming: Transcending STEM identity development through Afropessimism, moving toward a Black X consciousness in STEM. The Journal of Negro Education, 88(3), $327-342$.

Motala, S. \& Stewart, K. (2021). Hauntings across the divide: transdisciplinary activism, dualisms, and the ghosts of racism in engineering and humanities education. Canadian Journal of Science, Mathematics, and Technology Education. https://doi.org/10.1007/s42330-021-00153-7

Nxumalo, F. (2021). Disrupting anti-Blackness in early childhood qualitative inquiry: Thinking with Black refusal and Black futurity. Qualitative Inquiry.

Nxumalo, F. \& Ross, K.M. (2019). Envisioning Black space in environmental education for young children. Race Ethnicity \& Education, 22(4), 502-524.

Ortolano, N. (2020). 'We do belong here': The scientist behind \#BlackInNeuro hopes to transform a Twitter movement into a lasting community. Retrieved from: https://www.statnews.com/2020/08/26/we-do-belong-here-the-scientistbehind-blackinneuro-hopes-to-transform-a-twitter-movement-into-a-lasting-community/

Riegle-Crumb, C., King, B., \& Irizarry, Y. (2019). Does STEM stand out? Examining racial/ethnic gaps in persistence across postsecondary fields. Educational Researcher, 48, 133-144.

Ross, K.M. (2021). Anti-Blackness in education and the possibilities of redress: Toward educational reparations. Amerikastudien/American Studies, 66(1), 229-233.

Saddler, N., Adams, S., Robinson, L. A., \& Okafor, I. (2021). Taking initiative in addressing diversity in medicine. Canadian Journal of Science, Mathematics, and Technology Education. https://doi.org/10.1007/s42330-021-00154-6

Tuck, E. (2009). Suspending damage: A letter to communities. Harvard Educational Review, 79(3), 409-428.

Vakil, S., \& Ayers, R. (2019). The racial politics of STEM education in the USA: Interrogations and explorations. Race Ethnicity and Education, 22, 449-458.

Vossoughi, S., \& Vakil, S. (2018). Toward what ends? A critical analysis of militarism, equity, and STEM education. In A.I. Ali \& T. L. Buenavista, T. L. (Eds.) Education at war: The fight for students of color in America's public schools (pp. 117-140). Fordham University Press.

Warren, B., Vossoughi, S., Rosebery, A. S., Bang, M., Taylor, E. V. (2020). Multiple ways of knowing: Re-imagining disciplinary learning. In N.S. Nasir, C.D. Lee, R. Pea \& McKinney de Royston, M. (Eds.) Handbook of the cultural foundations of learning (pp. 277-293). Routledge.

Warren, C. A. (2021). From morning to mourning: A meditation on possibility in Black education. Equity \& Excellence in Education, 54(1), 92-102.

Wilderson, F. III. (2010). Red, white and black: Cinema and the structure of U.S. antagonisms. Duke University Press.

Wright, M. M. (2004). Becoming Black: Creating identity in the African diaspora. Duke University Press.

Publisher's Note Springer Nature remains neutral with regard to jurisdictional claims in published maps and institutional affiliations. 